\title{
Cerebral venous thrombosis after COVID-19 vaccination: the role for endovascular treatment
}

\author{
Amit Mahajan, ${ }^{1}$ Joshua A Hirsch (1) ${ }^{2}$
}

Cerebral venous thrombosis (CVT) is a unique cause of stroke. Population-based studies demonstrate an incidence of clinically significant CVT of $1.3-1.5$ per 100 000 per year. $^{12}$ This may be an underestimated incidence, as these studies were not based on review of imaging and the often benign nature of this entity may lead to underdiagnoses.

In the JNIS paper published by Cleaver et $a l,{ }^{3}$ the authors describe their experience with endovascular treatment (EVT) in patients with cerebral venous sinus thrombosis in patients following COVID-19 vaccination.

CVT-related stroke primarily affects young adult and middle-aged patients. ${ }^{4} 5$ Although initially blamed for having a 50\% mortality based on autopsy series, ${ }^{6}$ more recent prospective multinational studies (International Study on Cerebral Vein and Dural Sinus Thrombosis (ISCVT)) have demonstrated a mortality rate of $8 \%$ in a cohort of 624 patients. $^{7}$ A total of $75 \%$ of these patients were female and 13\% had a poor outcome (death or dependence). The baseline risk factors for poor outcome included age $>37$ years $(\mathrm{HR}=2.0)$, male sex $(H R=1.6)$, coma $(H R=2.7)$, mental status disorder $(\mathrm{HR}=2.0)$, hemorrhage on admission computed tomography (CT) scan $(H R=1.9)$, thrombosis of the deep cerebral venous system $(\mathrm{HR}=2.9)$, central nervous system infection $(\mathrm{HR}=3.3)$, and cancer $(\mathrm{HR}=2.9)$. Only $8 \%$ of these patients were $>65$ years of age, and had a poorer prognosis. ${ }^{8}$ Female predilection was lost in the elderly age group, with both sexes affected almost equally. ${ }^{5}$ Some $46 \%$ of these patients demonstrated infarct on $\mathrm{CT} / \mathrm{magnetic}$ resonance imaging (MRI) while $39 \%$ of these patients demonstrated hemorrhage.

Traditionally, all major guidelines ${ }^{9}$ have recommended anticoagulation with heparin - preferentially low molecular weight heparin - as the standard treatment

\section{Department of Radiology and Biomedical Imaging, Yale University, New Haven, Connecticut, USA 2Department of Radiology, Massachusetts General Hospital, Boston, Massachusetts, USA}

Correspondence to Dr Amit Mahajan, Department of Radiology and Biomedical Imaging, Yale University, New Haven, Connecticut, USA; amit.mahajan@yale.edu for CVT, regardless of the presence of an intracerebral hemorrhage (ICH). Heparin treatment is supposed to prevent growth or embolization of the existing thrombus, and facilitate recanalization. Patients with cerebral sinus thrombosis treated with anticoagulants (low molecular weight heparin followed by oral anticoagulation) had a favorable outcome more often than controls, although the difference was not statistically significant. ${ }^{10}$

Hypercoagulability and CVT after COVID-19 infection $^{11-13}$ and vaccination $^{14}$ has been described, especially after vaccination with the adenovirus vector vaccine ChAdOx1 (Oxford/AstraZeneca), which is associated with very low platelets, antiplatelet factor 4 antibodies and elevated d-dimer, leading to the designation of vaccine-induced thrombotic thrombocytopenia (VITT).

In a study of 95 patients with CVT, ${ }^{14}$ 70 of whom had VITT and 25 did not, primary outcome of death or dependency occurred more frequently in patients with VITT-associated CVT (47\%) compared with the non-VITT control group (16\%; $\mathrm{P}=0 \cdot 0061)$. The median age of the VITT group (47 years) was lower than in the non-VITT group (57 years; $\mathrm{P}=0 \cdot 0045$ ). Patients with VITT-associated CVT had more thrombosed intracranial veins (three) than non-VITT patients (two) and more frequently had extracranial thrombosis (44\%) compared with non-VITT patients (4\%; $\mathrm{P}=0.0003)$. The adverse outcomes were less frequent in patients with VITT who received non-heparin anticoagulants (18/50 (36\%) patients) compared with those who did not $(15 / 20$ (75\%) patients; $\mathrm{P}=0.0031)$, and in those who received intravenous immunoglobulin (22/55 (40\%) patients) compared with those who did not $(11 / 15(73 \%)$ patients; $\mathrm{P}=0 \cdot 022)$. Treatment guidelines advocate not using heparin in patients with suspected VITT; instead treatment is focused on intravenous immunoglobulin and nonheparin anticoagulation. $^{15}$

EVT is advocated in patients with COVID-19 infection-related emergent large vessel occlusion (ELVO) ${ }^{16}$ and to treat patients with CVT. ${ }^{17-20}$ EVT for CVT aims to achieve rapid recanalization of the cerebral venous sinuses through local mechanical thrombectomy, thrombolytic drug administration, or a combination of both. These methods result in more rapid recanalization, by delivering the drug where needed, downstream from the cerebral venous infarcts which may be hemorrhagic, and increasing the surface area of the thrombus exposed to thrombolytics. ${ }^{21}$ This is usually advocated when clinical neurologic deterioration occurs despite anticoagulation treatment.

The European Stroke Organisation guidelines, however, do not include or provide a recommendation on EVT for $\mathrm{CVT}^{9}{ }^{9}$ whereas the American Heart Association guideline recommended considering EVT only in patients who deteriorate despite anticoagulant treatment. ${ }^{5}$ In a systematic review of mechanical thrombectomy in CVT, of 185 cases $^{17}$ in 42 studies, $156(84 \%)$ of patients had a good outcome and 22 died (12\%). Some 10\% of patients demonstrated new or increased periprocedural hemorrhage with a failure rate of $5 \%$.

The TO-ACT (Thrombolysis or Anticoagulation for Cerebral Venous Thrombosis) trial $^{22}$ was designed to test the hypothesis that EVT in addition to standard medical care improves the clinical outcome of patients with severe CVT compared with standard medical care alone. Of 67 enrolled and randomized patients, 33 (49\%) were randomized to EVT and standard medical therapy (intervention group) and 34 (51\%) were randomized to receive guidelinebased standard medical care (control group). The intervention consisted of mechanical thrombectomy, pharmacological thrombolysis, or a combination of both. The interventional treatment was made at the discretion of the interventionalist, although the maximum doses of pharmacological agents were specified in the trial protocol. Continuous infusion with a thrombolytic drug in the affected sinus was allowed after the initial procedure for a maximum of 72 hours. For mechanical thrombectomy, the use of devices was allowed at the discretion of the interventionalist. At the 12-month follow-up, 22 intervention patients $(67 \%)$ had a modified Rankin score (mRS) of 0 to 1 compared with 23 control patients (68\%) (relative risk ratio 0.99). EVT with standard medical care did not appear to improve functional outcome of patients with CVT, although mortality was not statistically significantly higher in the EVT group (12\% vs $3 \% ; \mathrm{P}=0.20)$. The frequency of symptomatic ICH was also not statistically 
significantly lower in the intervention group ( $3 \%$ vs 9\%; $\mathrm{P}=0.61$ ). The study included adult patients (aged $\geq 18$ years) who had radiologically confirmed CVT with a high probability of poor outcome, defined as the presence of at least one of the following risk factors: mental status disorder, coma state (Glasgow Coma Scale score $<9$ ), ICH, or thrombosis of the deep cerebral venous system.

Considering the difference in outcomes and pathogenesis, and a higher mortality rate in this subgroup, a case for EVT can be made in this specific subgroup of patients with CVT. Although enticing, the use of EVT in this subgroup is likely to be of limited clinical relevance broadly, considering the low rate of vaccination with this type of vaccine (Johnson \& Johnson (JNJ) vaccine 16 million vs 172 million for Moderna and 263 million for the Pfizer vaccine). ${ }^{23}$ Given the relative infrequency of this complication, the low rate of vaccination of world populations using the JNJ vaccine, and the high relative costs of EVT, this is likely to remain a niche therapy to be used in the direst of circumstances, to be used if local neurointerventional expertise is available.

Whether the results of this study should open up the use of EVT to all patients with CVT is a large leap of faith, based on the negative results of the TO-ACT trial. Although a case for EVT could still be made, based on the heterogeneity of the study population and the lack of standardization of EVT therapy in this particular condition across numerous worldwide sites, the relative value of this technique still needs to be established in a well-designed, and perhaps larger, clinical trial under controlled conditions.

\section{Twitter Amit Mahajan @Amit_Yale and Joshua A Hirsch @JoshuaAHirsch}

Contributors Both authors contributed equally to this commentary.

Funding The authors have not declared a specific grant for this research from any funding agency in the public, commercial or not-for-profit sectors.

Competing interests None declared.

Patient consent for publication Not applicable.

Ethics approval This study does not involve human participants.
Provenance and peer review Not commissioned; externally peer reviewed.

Data availability statement Data sharing not applicable as no datasets generated and/or analyzed for this study. Not applicable.

Supplemental material This content has been supplied by the author(s). It has not been vetted by BMJ Publishing Group Limited (BMJ) and may not have been peer-reviewed. Any opinions or recommendations discussed are solely those of the author(s) and are not endorsed by BMJ. BMJ disclaims all liability and responsibility arising from any reliance placed on the content. Where the content includes any translated material, BMJ does not warrant the accuracy and reliability of the translations (including but not limited to local regulations, clinical guidelines, terminology, drug names and drug dosages), and is not responsible for any error and/or omissions arising from translation and adaptation or otherwise.

(c) Author(s) (or their employer(s)) 2022. No commercial re-use. See rights and permissions. Published by BMJ.

- Additional supplemental material is published

online only. To view, please visit the journal online (http://dx.doi.org/10.1136/neurintsurg-2021018516).

\section{Check for updates}

To cite Mahajan A, Hirsch JA. J Neurolntervent Surg Epub ahead of print: [please include Day Month Year]. doi:10.1136/neurintsurg-2021-018516

Accepted 30 January 2022

J Neurolntervent Surg 2022;0:1-2.

doi:10.1136/neurintsurg-2021-018516

\section{ORCID iD}

Joshua A Hirsch http://orcid.org/0000-0002-9594-8798

\section{REFERENCES}

1 Coutinho JM, Zuurbier SM, Aramideh M, et al. The incidence of cerebral venous thrombosis. Stroke 2012:43:3375-7.

2 Devasagayam S, Wyatt B, Leyden J, et al. Cerebral venous sinus thrombosis incidence is higher than previously thought. Stroke 2016;47:2180-2.

3 Cleaver J, Ibitoye R, Morrison H, et al. Endovascular treatment for vaccine-induced cerebral venous sinus thrombosis and thrombocytopenia following ChAdOx1 nCoV-19 vaccination: a report of three cases. $J$ Neurointerv Surg 2021:neurintsurg-2021-018238.

4 Silvis SM, de Sousa DA, Ferro JM, et al. Cerebral venous thrombosis. Nat Rev Neurol 2017;13:555-65.

5 Saposnik G, Barinagarrementeria F, Brown RD, et al. Diagnosis and management of cerebral venous thrombosis. Stroke 2011;42:1158-92.

6 Stam J. Cerebral venous and sinus thrombosis: incidence and causes. Adv Neurol 2003;92:225-32.

7 Ferro José M., Canhão Patrícia, Stam J, et al. Prognosis of cerebral vein and dural sinus thrombosis. Stroke 2004;35:664-70.
8 Ferro JM, Canhão P, Bousser M-G, et al. Cerebral vein and dural sinus thrombosis in elderly patients. Stroke 2005;36:1927-32.

9 Ferro JM, Bousser M-G, Canhão P, et al. European Stroke Organization guideline for the diagnosis and treatment of cerebral venous thrombosis - endorsed by the European Academy of Neurology. Eur J Neurol 2017:24:1203-13.

10 de Bruijn SF, Stam J, SFTMd B. Randomized, placebocontrolled trial of anticoagulant treatment with low-molecular-weight heparin for cerebral sinus thrombosis. Stroke 1999;30:484-8.

11 Cavalcanti DD, Raz E, Shapiro M, et al. Cerebral venous thrombosis associated with COVID-19. AJNR Am J Neuroradiol 2020;41:1370-6.

12 Moonis G, Filippi CG, Kirsch CFE, et al. The spectrum of neuroimaging findings on $\mathrm{CT}$ and MRI in adults with COVID-19. AJR Am J Roentgenol 2021;217:959-74.

13 Zakeri A, Jadhav AP, Sullenger BA, et al. Ischemic stroke in COVID-19-positive patients: an overview of SARS-CoV-2 and thrombotic mechanisms for the neurointerventionalist. J Neurointerv Surg 2021:13:202-6.

14 Perry RJ, Tamborska A, Singh B, et al. Cerebral venous thrombosis after vaccination against COVID-19 in the UK: a multicentre cohort study. Lancet 2021;398:1147-56.

15 Rizk JG, Gupta A, Sardar P, et al. Clinical characteristics and pharmacological management of COVID-19 vaccine-induced immune thrombotic thrombocytopenia with cerebral venous sinus thrombosis: a review. JAMA Cardiol 2021;6:1451.

16 Fiorella D, Fargen KM, Leslie-Mazwi TM, et al. Neurointervention for emergent large vessel occlusion during the COVID-19 pandemic. J Neurointerv Surg 2020;12:537-9.

17 Siddiqui FM, Dandapat S, Banerjee C, et al. Mechanical thrombectomy in cerebral venous thrombosis. Stroke 2015:46:1263-8

18 Fraser JF, Arthur AS, Chen M, et al. Society of Neurolnterventional Surgery recommendations for the care of emergent neurointerventional patients in the setting of COVID-19. J Neurointerv Surg 2020;12:539-41.

19 Aggour M, White P, Kulcsar Z, et al. European Society of Minimally Invasive Neurological Therapy (ESMINT) recommendations for optimal interventional neurovascular management in the COVID-19 era. J Neurointerv Surg 2020;12:542-4.

20 Yang B, Wang T, Chen J, et al. Impact of the COVID-19 pandemic on the process and outcome of thrombectomy for acute ischemic stroke. J Neurointerv Surg 2020;12:664-8.

21 Stam J, Majoie CBLM, van Delden OM, et al. Endovascular thrombectomy and thrombolysis for severe cerebral sinus thrombosis: a prospective study. Stroke 2008;39:1487-90.

22 Coutinho JM, Zuurbier SM, Bousser M-G, et al. Effect of endovascular treatment with medical management vs standard care on severe cerebral venous thrombosis: the TO-ACT randomized clinical trial. JAMA Neurol 2020;77:966-73.

23 Centers for Disease Control and Prevention. COVID data tracker: COVID-19 vaccinations in the United States, 2022. Available: https://covid.cdc.gov/coviddata-tracker/\#vaccinations_vacc-total-admin-ratetotal 